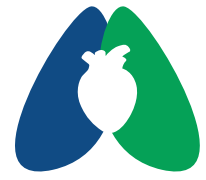

ASSOBRAFIR C I Ê N C I A

\section{Impacto da reabilitação pulmonar na função respiratória e capacidade funcional em paciente com paralisia diafragmática unilateral: relato de caso}

\author{
Impact of pulmonary rehabilitation on respiratory function and \\ functional capacity in a patient with unilateral diaphragmatic \\ paralysis: a case report
}

\begin{abstract}
Rafaela Correia de Souza Cunha ${ }^{1}$ (D); Rafaela Pedrosa ${ }^{1}$; ; Andrezza Soares Dias ${ }^{1}$ (D); Carlos Eduardo Porto da Silva² (1D; Eduardo Eriko Tenório de França ${ }^{1}$ (D); José Heriston de Morais Lima1* (D)

${ }^{1}$ Universidade Federal da Paraíba (UFPB), João Pessoa, PB, Brasil ${ }^{2}$ Hospital Universitário Lauro Wanderley, João Pessoa, PB, Brasil

Como citar: Cunha RCS, Pedrosa R, Dias AS, Silva CEP, França EET, Lima JHM. Impacto da reabilitação pulmonar na função respiratória e capacidade funcional em paciente com paralisia diafragmática unilateral: relato de caso. ASSOBRAFIR Ciênc. 2020;11:e41229. https://doi.org/10.47066/2177-9333.AC.2020.0007
\end{abstract}

\begin{abstract}
Resumo
Introdução: a reabilitação pulmonar (RP) foi definida como um programa multidisciplinar de atendimento a pacientes com insuficiência respiratória crônica, individualmente adaptado e projetado para otimizar o desempenho físico e social e a autonomia. Objetivo: identificar a repercussão da RP na função respiratória, capacidade funcional e na sensação de dispneia em um paciente com paralisia diafragmática unilateral. Metodologia: trata-se de um relato de caso. Paciente do sexo masculino, 51 anos, com diagnóstico de paralisia diafragmática unilateral foi submetido durante dois meses a um protocolo de RP composto por exercícios aeróbios, treinamento muscular respiratório (PowerBreathe IMT) e fortalecimento de membros superiores. Foram avaliados a força e resistência muscular respiratória, função pulmonar, capacidade funcional e percepção de dispneia. Resultados: o paciente apresentou um aumento da capacidade vital forçada em $24,1 \%$ e do volume expiratório forçado no primeiro segundo em $26,4 \%$. Houve um ganho de $29,7 \%$ na força muscular respiratória e um aumento de $38,5 \%$ no tempo da execução do teste de resistência com um aumento de $33,3 \%$ na carga suportada durante este teste, elevação de $6 \%$ na velocidade da esteira durante o teste de esforço e um aumento de $40 \%$ na carga do teste incremental de membros superiores. Além disso, a percepção de dispneia do paciente diminuiu de 4 para 1. Conclusão: os resultados obtidos sugerem que a RP contribuiu para melhora da função pulmonar e músculo ventilatória, como também, apresentou-se eficiente na melhora da capacidade funcional e na redução da dispneia do paciente diagnosticado com paralisia diafragmática unilateral.
\end{abstract}

Palavras-chave: Reabilitação; Paralisia Respiratória; Mecânica Respiratória.

\begin{abstract}
Background: Pulmonary rehabilitation (PR) was defined as a multidisciplinary care program for patients with chronic respiratory failure, individually adapted and designed to optimize physical and social performance and autonomy. Aim: to identify the repercussions of PR on respiratory function, functional capacity and the sensation of dyspnea in a patient with unilateral diaphragmatic paralysis. Methods: this is a case report. A 51-year-old male patient, diagnosed with unilateral diaphragmatic paralysis, underwent a PR protocol consisting of aerobic exercises, respiratory muscle training (PowerBreathe IMT) and strengthened upper limbs for two months. Respiratory muscle strength and endurance, lung function, functional capacity and perception of dyspnea were evaluated. Results: the patient presented an increase in the forced vital capacity in $24.1 \%$ and in the forced expiratory volume in the first second in $26.4 \%$. There was a gain of $29.7 \%$ in respiratory muscle strength and an increase of $38.5 \%$ in the time of execution of the resistance test with an increase of $33.3 \%$ in the
\end{abstract}

Estudo realizado em: Hospital Universitário Lauro Wanderley, Universidade Federal da Paraíba, João Pessoa, PB, Brasil. Aprovação ética: CAEE 86468218.6.0000.5183 do Hospital Lauro Wanderley da Universidade Federal da Paraíba, $n^{\circ}$ 2.656.106.

*Autor correspondente: José Heriston de Morais Lima. E-mail: joseheristonlima@yahoo.com.br of $38.5 \%$ in the time of execution of the resistance test with an increase of $33.3 \%$ in the sob a mesma licença 
load supported during this test, $6 \%$ increase in the speed of treadmill during the stress test and a $40 \%$ increase in the load of the incremental upper limb test. In addition, the patient's perception of dyspnea decreased from 4 to 1 . Conclusion: the results obtained suggest that PR contributed to improve pulmonary function and ventilatory muscle, as well as being efficient in improving functional capacity and reducing blood pressure. dyspnea of the patient diagnosed with unilateral diaphragmatic paralysis.

Keywords: Rehabilitation; Respiratory Paralysis; Respiratoy Mechanics.

\section{INTRODUÇÃO}

A fraqueza diafragmática é a perda parcial da força muscular para gerar a pressão necessária para uma ventilação adequada, enquanto paralisia significa a total ausência dessa capacidade. Esse distúrbio, dependendo da causa, pode ser unilateral ou bilateral, temporário ou permanente. A paralisia diafragmática idiopática é a etiologia mais frequente (50\%). A condição geralmente é subdiagnosticada e há poucos dados epidemiológicos disponíveis, exceto lesões traumáticas pós-cirúrgicas ${ }^{1}$. $\mathrm{Na}$ radiografia de tórax, eles serão observados como elevação localizada do diafragma².

Como o diafragma é o principal músculo respiratório, sua disfunção pode estar associada à presença de sintomas respiratórios, intolerância ao exercício, distúrbios do sono e, nos casos mais graves, ter um impacto negativo na sobrevida. O diagnóstico e o manejo uni e bilateral da disfunção do diafragma podem ser problemáticos para o clínico devido à sua relativa raridade, às suas manifestações clínicas às vezes sutis e às dificuldades na obtenção de um diagnóstico fisiologicamente confirmado ${ }^{3}$.

A American Thoracic Society em seu Guideline sobre Reabilitação Pulmonar (RP), atesta que a RP melhora a capacidade para o exercício, reduz a dispneia, melhorando a qualidade de vida, ajudando a restaurar a função emocional, reduzindo os níveis de depressão e ansiedade e aumentando a capacidade do paciente em controlar a própria doença ${ }^{4}$. Diante do exposto, $\mathrm{O}$ objetivo do estudo foi identificar a repercussões da reabilitação pulmonar, na função respiratória, capacidade funcional e na sensação de dispneia em um paciente com paralisia diafragmática unilateral.

\section{MÉTODOS}

O estudo foi caracterizado como relato de caso, o paciente foi encaminhado do serviço de pneumologia do Hospital Lauro Wanderley da Universidade Federal da Paraíba - UFPB, com diagnóstico de paralisia diafragmática unilateral e sinais de desvio contralateral das estruturas mediastinais decorrente de acidente automobilístico há aproximadamente oito anos, apresentando como queixa principal dispneia aos esforços. No ambulatório de Fisioterapia do mesmo hospital, foi admitido no Projeto de extensão em Reabilitação pulmonar após assinar o Termo de Consentimento Livre e Esclarecido. O estudo foi aprovado pelo Comitê de Ética em Pesquisa do Hospital Lauro Wanderley da UFPB, sob o número
CAE: 86468218.6.0000.5183, respeitando a Resolução 466/2012 do Conselho Nacional de Saúde do Brasil.

Para avaliação da função muscular respiratória foi utilizado o dispositivo eletrônico e computadorizado (KH2; PowerBreathe International Ltd. UK) com o Software de feedback Breathelink, sendo avaliado a pressão inspiratória máxima (maximum inspiratory pressure - MIP) e a resistência da musculatura respiratória foi avaliada pelo teste de carga incremental utilizando o mesmo aparelho. Foi padronizado uma carga inicial de $10 \mathrm{cmH}_{2} \mathrm{O}$ por um período de 2 minutos. Posteriormente o paciente foi orientado a descansar por 1 minuto e em seguida, um novo ciclo de 2 minutos foi iniciado, acrescendo mais $10 \mathrm{cmH}_{2} \mathrm{O}$ a carga do equipamento. A maior carga sustentada por pelo menos 1 minuto foi considerada o valor da MIP sustentada (SMIP). A MIP foi avaliada antes e imediatamente após o teste, a fim de verificar a ocorrência de fadiga muscular ${ }^{5,6}$.

A avaliação da função pulmonar foi realizada no espirômetro digital Pneumotach DATALINK, sendo avaliado capacidade vital forçada (CVF); volume expiratório forçado no primeiro segundo $\left(V_{E F}\right)$ e índice de Tiffeneau (VEF $/$ /CVF). Para avaliação da capacidade funcional foi realizado o teste de esforço incremental em esteira ergométrica e a força da musculatura periférica foi realizado o teste incremental para membros superiores. A sensação de dispneia foi avaliada pela Medical Research Council - MRC e escala de percepção de esforço de Borg7-9.

O protocolo de reabilitação pulmonar foi composto por exercícios aeróbios realizados em esteira ergométrica, aumentando a velocidade e o tempo de exercício semanalmente através da Escala de Percepção de Esforço de Bog e utilizando a escala de dispnéia de Medical Research Council (MRC) para interrupção e segurança do paciente durante a atividade. $O$ treinamento muscular respiratório foi realizado com o dispositivo PowerBreathe IMT com carga de $30 \mathrm{cmH}_{2} \mathrm{O}$ realizando três séries de dez repetições e exercício resistido de membros superiores, através de bastão com caneleiras de vários pesos que eram trocadas de acordo com a escala de percepção de esforço de Borg.

\section{RESULTADOS}

O Paciente M.A.P.A., 51 anos, sexo masculino, diabético, hipertenso, sobrepeso com índice de massa corpórea de vinte e sete, com diagnóstico de paralisia diafragmática unilateral e sinais de desvio contralateral das estruturas mediastinais, tendo como queixa principal dispneia aos esforços, sendo avaliado no dia 07/07/19, iniciado o 
tratamento no dia 09/07/19 até 19/09/19 totalizando dois messes de atendimento. As sessões eram realizadas duas vezes por semana.

Na avaliação no teste incremental de esforço na esteira o paciente alcançou uma velocidade máxima de $5 \mathrm{Km} / \mathrm{h}$ com percepção de esforço de Borg de 10. No teste de resistência dos músculos respiratórios o paciente conseguiu realizar o treinamento em cinco minutos com carga final de $30 \mathrm{~cm} / \mathrm{H}_{2} \mathrm{O}$ e percepção de esforço de Borg de 7. No teste incremental de membros superiores o paciente conseguiu peso final de $5 \mathrm{Kg}$ com Borg de 8. Os resultados obtidos na avaliação espirométrica demostram um distúrbio ventilatório restritivo grave, caracterizado pela relação $V F_{1} / C V F$ normal, com redução da CVF, conforme Tabela 1 abaixo:

Quando analisamos indiretamente a força da musculatura inspiratória através da MIP encontramos o valor de $-53,88 \mathrm{cmH}_{2} \mathrm{O}$ (valor previsto -113,7 $\mathrm{cmH}_{2} \mathrm{O}$ ), evoluindo para $-69,88 \mathrm{cmH}_{2} \mathrm{O}$, um ganho de $29,7 \%$ na força muscular inspiratória, demonstrado na Figura 1.

Considerando a resistência dessa musculatura, houve uma evolução no tempo, passando de 5,20 min com de carga de $30 \mathrm{cmH}_{2} \mathrm{O}$ para 7,20 min com carga de $40 \mathrm{cmH}_{2} \mathrm{O}$ após tratamento, ocorrendo incremento de $38,5 \%$ no tempo de execução do teste e um aumento de $33,3 \%$ na carga suportada, quando comparados aos dados da avaliação inicial, também ocorreu melhora no teste incremental de membros superiores e na escala de dispneia.

Ao realizar o teste incremental de MMSS na primeira avaliação, o paciente conseguiu uma carga máxima de $5 \mathrm{Kg}$ com Borg relatado de 8, e na avaliação final alcançou uma carga de $7 \mathrm{Kg}$ com Borg de 8. Quando questionamos o paciente quanto a escala de dispneia (MRC), o mesmo relatou Grau 4 - "Muito cansado para sair de casa ou se vestir" evoluindo para Grau 1 - "Falta de ar quando caminha depressa no plano ou sobe ladeira leve" na avaliação final.

\section{DISCUSSÃO}

Neste estudo realizamos Reabilitação Pulmonar em um paciente com paralisia diafragmática unilateral com queixa de dispneia aos esforços e também, apresentava valores muito abaixo do previsto em sua avaliação respiratória. A reabilitação pulmonar melhora a tolerância ao exercício e tem como elemento fundamental o treinamento físico que, somado às demais estratégias, tem por objetivos melhorar e controlar os sintomas, minimizar as complicações da doença e auxiliar os pacientes a viver uma vida ativa com poucas restrições ${ }^{10}$.

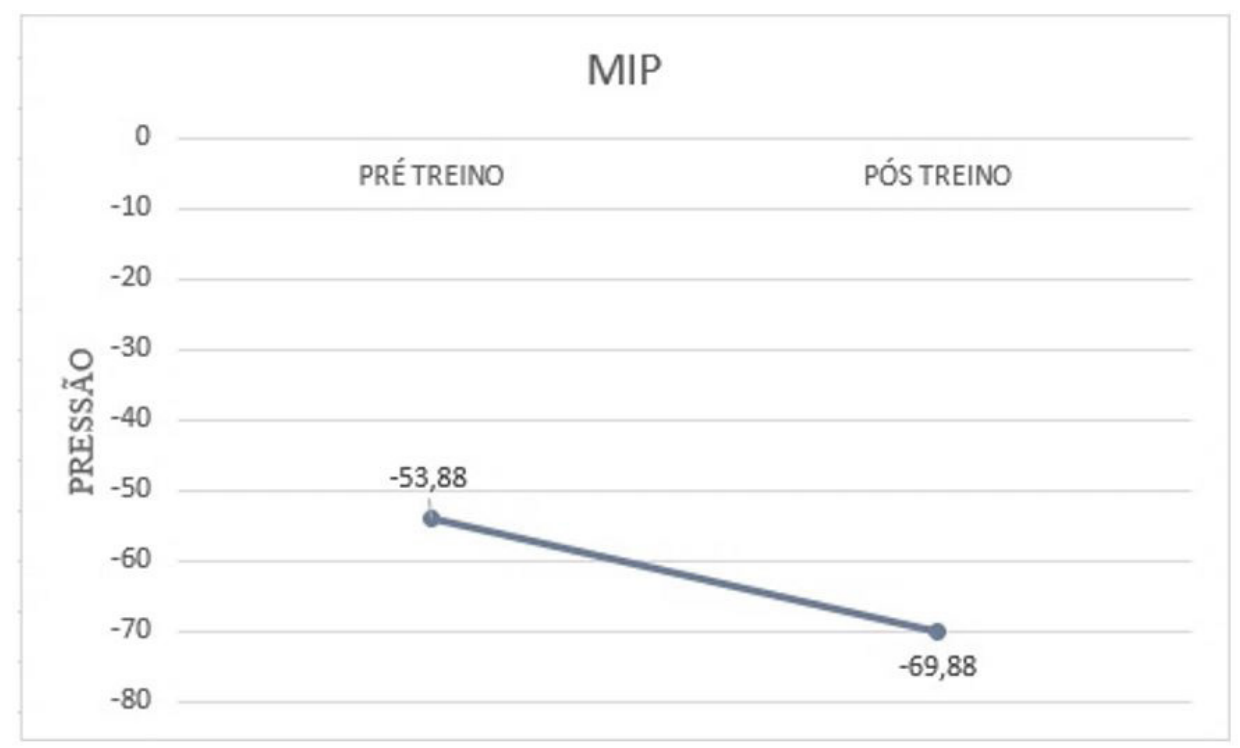

Figura 1. Valores de pressão inspiratória máxima (MIP) antes e após treino de reabilitação pulmonar.

Fonte: dados da pesquisa.

Tabela 1. Valores de Capacidade vital forçada (CVF); volume expiratório forçado no primeiro segundo $\left(V_{E} F_{1}\right)$ e índice de Tiffeneau (VEF1/CVF) pré e pós tratamento.

\begin{tabular}{ccccc}
\hline VARIÁVEIS & Valor predito & Valor pré tratamento & Valor pós tratamento & Ganho (\%) \\
\hline CVF $(\mathrm{L})$ & 3,81 & 0,58 & 0,72 & 24,1 \\
VEF $_{1}(\mathrm{~L})$ & 3,10 & 0,53 & 0,67 & 26,4 \\
VEF $_{1}$ /CVF & 81,2 & 90,1 & 93,1 & 3,0
\end{tabular}

Fonte: Dados da pesquisa. 
O movimento abdominal paradoxal pode ocorrer em pacientes com paralisia diafragmática, o que sugere que nesses pacientes, o movimento abdominal paradoxal está relacionado a uma ventilação fisiologicamente ineficiente resultado da ascensão do diafragma no lado afetado restringindo o fluxo de ar inspiratório, explicando a perda da capacidade de exercício em pacientes com DP unilateral'.

Segundo Troosters et al. ${ }^{11}$, muito progresso foi feito na RP, particularmente na prescrição de exercícios, e formas alternativas de exercício foram introduzidas com sucesso além do treinamento convencional de alta intensidade, normalmente usados para melhorar a condição física. Devido ao efeito avassalador da RP, é importante que o serviço seja disponibilizado ao maior número possível de pacientes necessitados. Isso requer a necessidade de programas que estejam dentro do alcance de pacientes e médicos que se referem adequadamente a esses programas.

Foi demonstrado também que o treinamento muscular inspiratório na RP aumenta a proliferação de fibras do tipo 1 e fibras musculares inspiratórias do tipo 2 , resultando em benefícios de resistência respiratória em pacientes com DPOC após 8 semanas de treinamento, após 11 semanas de treinamento em atletas e após 4 semanas em pessoas sedentárias ${ }^{12}$. O resistor linear pressórico foi capaz de alcançar padrões de ativação na Eletroneuromiografia semelhantes com ativação diafragmática efetiva. No geral, os músculos acessórios respiratórios e o diafragma atingiram níveis mais altos de ativação neuromuscular, quando utilizado o treinamento muscular respiratório com o resistor linear pressórico ${ }^{13}$

A principal limitação deste estudo é ser relato de um único caso, já que isso limita na extrapolação do uso dos resultados para a prática clínica, porém, é uma primeira evidência na importância da reabilitação pulmonar em sujeitos com paralisia diafragmática. Um ensaio clínico controlado para testar o tamanho do efeito desta intervenção nesta mesma população deve ser conduzido para respaldar a indicação na prática clínica.

\section{CONCLUSÃO}

Os resultados obtidos sugerem que a reabilitação pulmonar contribui para melhora da função pulmonar e músculoventilatória, como também, apresenta-se eficiente na melhora da capacidade funcional e na redução da dispnéia do paciente diagnosticado com paralisia diafragmática unilateral.

\section{FONTE DE FINANCIAMENTO}

Nada a declarar.

\section{CONFLITO DE INTERESSES}

Nada a declarar.

\section{REFERÊNCIAS}

1. Bonnevie T, Gravier FE, Ducrocq A, Debeaumont D, Viacroze C, Cuvelier A, et al. Exercise testing in patients with diaphragm. Respir Physiol Neurobiol. 2018;248:31-5. http:// dx.doi.org/10.1016/j.resp.2017.11.006. PMid:29155335.

2. Ricoy J, Rodríguez-Núñez N, Álvarez-Dobaño JM, Toubes ME, Riveiro V, Valdés L. Diaphragmatic dysfunction. Pulmonology. 2019;25(4):223-35. http://dx.doi.org/10.1016/j. pulmoe.2018.10.008. PMid:30509855.

3. Dubé BP, Dres M. Diaphragm dysfunction: diagnostic approaches and managent strategies. J Clin Med. 2016;5(12):1-30.

4. Ribeiro BV. Reabilitação pulmonar: da teoria à prática. Pulmão. 2015;24(3):54-8.

5. POWERbreathe International Ltd. POWERbreathe $\mathrm{KH} 2$ with Breathe-Link medic live feedback software. England, UK; 2020.

6. Sales ATN, Fregonezi GAF, Ramsook AH, Guenette JA, Lima INDF, Reid WD. Respiratory muscle endurance fter respiratory muscle training in athletes and non-athletes: a systematic review and meta-analysis. Phys Ther Sport. 2016;17:76-86.

7. Pereira CA, Sato T, Rodrigues SC. Novos valores de referencia para espirometria forçada em Brasileiros adultos de raça branca. J Bras Pneumol. 2007;33(4):397-406. http://dx.doi. org/10.1590/S1806-37132007000400008. PMid:17982531.

8. Laghi F, Tobin MJ. Disorders of the respiratory muscles. Am J Respir Crit Care Med. 2003;168(1):10-48.

9. Cabral LL, Lopes PB, Wolf R, Stefanello JMF, Pereira G. Revisão sistemática da adaptação transcultural e validação da escala de percepção de esforço de borg. J Phys Educ. 2017;28:1-13.

10. Evans RA, Singh SJ, Collier R, Williams JE, Morgan MD. Pulmonary rehabilitation is successful for COPD irrespective of MRC dyspnoea grade. Respir Med. 2009;103(7):10705. http://dx.doi.org/10.1016/j.rmed.2009.01.009. PMid:19217765.

11. Troosters T, Blondeel A, Janssens W, Demeyer H. The past, presente and future of pulmonar rehabilitation. Resprology. 2019;24(9):830-7. http://dx.doi.org/10.1111/resp.13517.

12. Bissett BM, Leditschke IA, Neeman T, Boots R, Paratz $J$. Inspiratory muscle training to enhance recoverry from machanical ventilation: a randomised trial. Thorax. 2016;71(9):812-9. http://dx.doi.org/10.1136/ thoraxjnl-2016-208279. PMid:27257003.

13. Walterspacher S, Pietsch F, Walker DJ, Röcker K, Kabitz HJ. Activation of respiratory muscles during respiratory muscle training. Respir Physiol Neurobiol. 2018;247:126-32. http:// dx.doi.org/10.1016/j.resp.2017.10.004. PMid:29037769. 